\title{
Der Vertrag von Prüm: Vertiefungs- und Fragmentierungs- tendenzen in der Justiz- und Innenpolitik der EU
}

\author{
Daniela Kietz und Andreas Maurer*
}

Der am 27. Mai 2005 in Prüm in der Eifel unterzeichnete und auf Initiative Deutschlands verhandelte Vertrag über die Vertiefung der grenzüberschreitenden Zusammenarbeit, zur Bekämpfung des Terrorismus, der Kriminalität und der illegalen Migration zwischen Deutschland, Österreich, den Benelux-Staaten, Spanien und Frankreich (VPgZ) ist eine Initiative zur Vertiefung der polizeilichen Zusammenarbeit in der Europäischen Union (EU). ${ }^{1}$ Der Vertrag befindet sich gegenwärtig im Prozess der Ratifizierung durch die nationalen Parlamente. ${ }^{2}$ Bereits jetzt haben etliche Staaten Interesse an einem Beitritt geäußert, der Beitritt Finnlands noch in diesem Jahr ist sehr wahrscheinlich. ${ }^{3}$ Nach dem Vorbild der Schengen-Kooperation, dem Schengener Übereinkommen (SÜ) und dem Schengener Durchführungsübereinkommen (SDÜ) aus den Jahren 1985 und 1990 handelt es sich bei diesem Vertrag um eine Art der verstärkten Zusammenarbeit zwischen sieben EU-Mitgliedstaaten außerhalb des EU-Vertragsrahmens. ${ }^{4}$ Analog zur Schengen-Kooperation verfolgt der Vertrag das Ziel später in den EU-Rahmen überführt zu werden. Das Schengener Vertragswerk wurde im Jahr 1999 durch den Amsterdamer Vertrag in den EU-Rechtsrahmen überführt. Auch wenn eine Reihe von Mitgliedstaaten heute noch Ausnahmeregelungen hinsichtlich ihrer Teilnahme am Schengen Acquis in Anspruch nehmen, wird der Schengener Integrationsprozess als Motor der Entwicklung der europäischen Innen- und Justizpolitik betrachtet; Prüm soll eben diese Vorreiterrolle für den Bereich der polizeilichen Zusammenarbeit übernehmen. ${ }^{5}$ Im Vergleich mit Schengen wirft der Prümer Vertrag vor allem zwei Fragen auf:

Erstens, geht man aus Gründen der inneren und äußeren Handlungsfähigkeit der EU von dem mittlerweile im EU-Vertrag akkordierten Ziel der Schaffung eines einheitlichen, EUweiten Raumes der Freiheit, der Sicherheit und des Rechts aus, dann ist zu prüfen, ob der Prümer Vertrag diesem Ziel dient, oder ob er eher zu einer weiteren Fragmentierung der EU führt. Das Ausweichen der EU-Mitgliedstaaten in den kleineren, zwischenstaatlichen Verhandlungsrahmen lässt sich vor dem Hintergrund der vom Einstimmigkeitszwang im Ministerrat geprägten, mühsamen Entscheidungsfindung in der polizeilichen und justiziellen $\mathrm{Zu}-$

* Dipl.-Pol. Daniela Kietz, Forschungsgruppe EU-Integration, Stiftung Wissenschaft und Politik, Berlin. Dr. Andreas Maurer, Leiter der Forschungsgruppe EU-Integration, Stiftung Wissenschaft und Politik, Berlin.

1 Siehe den Vertragstext unter: www.parlinkom.gv.at/portal/page?_pageid=908,974671\&SUCHE=J\&_dad=por tal\&_schema=PORTAL\# (letzter Zugriff: 19.06.2006).

2 Bisher haben Österreich, Spanien und Deutschland den Vertrag ratifiziert.

3 Vorträge der Österreichischen Ratspräsidentschaft und des Finnischen Parlaments, Öffentliches Seminar zum Vertrag von Prüm, Europäisches Parlament, 22. Juni 2006.

4 Vgl. zum Vertrag auch Thierry Balzacq u.a.: Security and the Two Level Game: The Treaty of Prüm, the EU and the Management of Threats, CEPS Working Document Nr. 234/2006, abrufbar unter: www.ceps.be (letzter Zugriff: 19.06.2006); Daniela Kietz/Andreas Maurer: Von Schengen nach Prüm. Sogwirkungen verstärkter Kooperation und Anzeichen der Fragmentierung in der EU, SWP-Aktuell Nr.24/2006, abrufbar unter: www.swp-berlin.org (letzter Zugriff: 19.06.2006); Franklin Dehousse/Diane Sifflet: Les Nouvelles Perspectives de la Cooperation de Schengen: Le Traité de Prüm, Institut Royal des Relations Internationales, Occasional Papers, abrufbar unter: http://www.irri-kiib.be/papers_eur.htm (letzter Zugriff: 19.06.2006).

5 Präsentation des Vertragswerkes durch die österreichische Präsidentschaft in der Sitzung des Ausschuss für Bürgerliche Freiheiten, Justiz und Inneres des Europäischen Parlaments am 4. Mai 2006. Der Vertrag wird gelegentlich als ,Schengen III‘ oder, Schengen plus ‘ bezeichnet. 
sammenarbeit (PJZS) durchaus nachvollziehen. Die Befürworter erhoffen sich von dieser Vorgehensweise eine Intensivierung und Beschleunigung des bislang nur stockend verlaufenden Entwicklungsfortschritts. Nun haben sich allerdings die Rahmenbedingungen der europäischen Justiz- und Innenpolitik seit dem Beginn der Schengen-Kooperation stark verändert und damit auch die Bedingungen für eine Wiederholung des Schengen-Prozesses. Mittlerweile besteht zum einen ein umfassender Rechtskörper in der europäischen Innenund Justizpolitik. Zum anderen haben sich die Entscheidungsstrukturen auf europäischer Ebene seit Schengen stark verändert. Das Ziel des Prümer Vertrages, das Voranbringen der Integration in den betreffenden Politikbereichen, wird von allen Akteuren in der europäischen Innen- und Justizpolitik geteilt. Der Vertrag mag dieses Ziel auch erreichen. Zur Debatte stehen jedoch der gewählte Weg, nämlich das Ausweichen in den zwischenstaatlichen Raum, und der damit verbundene mögliche Preis einer weiteren Fragmentierung der Innenund Justizpolitik auf europäischer Ebene sowie das Umgehen der EU-Institutionen.

Zweitens, gilt es die demokratisch-parlamentarische Kontrolle des Prümer Prozesses kritisch zu prüfen ${ }^{6}$. Der Schengener Integrationsprozess wies diesbezüglich eine ganze Reihe an Mängeln auf. ${ }^{7}$ Wie schon Schengen vor ihm regelt Prüm höchst sensible Politikbereiche die im innenstaatlichen Rahmen normalerweise der intensiven Prüfung aus demokratie-, grund-, bürger- und datenschutzrechtlicher Sicht unterliegen. In der Begründung zum Ratifikationsgesetz des Deutschen Bundestages und des Bundesrates heißt es lapidar: „Die Grundrechte auf Leben und der körperlichen Unversehrtheit (Artikel 2 Abs. 2 Satz 1 des Grundgesetzes), der Freiheit der Person (Artikel 2 Abs. 2 Satz 2 des Grundgesetzes), der Freiheit der Versammlung (Artikel 8 des Grundgesetzes), des Brief-, Post- und Fernmeldegeheimnisses (Artikel 10 des Grundgesetzes) und der Unverletzlichkeit der Wohnung (Artikel 13 des Grundgesetzes) werden nach Maßgabe dieses Gesetzes eingeschränkt.“ Es stellt sich daher die Frage, was die ratifikationspflichtigen Parlamente diesbezüglich aus dem Schengener Integrationsprozess lernen können.

Im folgenden Abschnitt soll zunächst die Einbettung des Prümer Vertrages in den EURahmen dargelegt werden, um danach die eben aufgeworfenen Bewertungsmassstäbe einerseits der demokratischen Kontrolle des Vertrages und andererseits seiner Vereinbarkeit mit dem Ziel der Schaffung eines einheitlichen Raumes der Freiheit der Sicherheit und des Rechts anzulegen.

\section{Die Einbettung des Prümer Vertrags in den EU-Rahmen}

Seit dem Vertrag von Amsterdam ist die Schaffung eines einheitlichen EU-weiten Raums der Freiheit, der Sicherheit und des Rechts erklärtes Ziel der Europäischen Union (EU). Der Vertrag überführte zu diesem Zweck den Schengen-Acquis in den Rechtsrahmen der Union und sah zudem die schrittweise Überführung der Mehrzahl der justiz- und innenpolitischen Teilbereiche aus der intergouvernementalen Kooperation in der so genannten ,Dritten Säule in den supranationalen Gemeinschaftsrahmen vor. Mit Blick auf die Entscheidungsfindung bedeutete dies vor allem den effizienzfördernden Übergang von der Einstimmigkeit zu Mehrheitsentscheidungen im Ministerrat und aus demokratiepolitischer Sicht die stärkere

6 Vgl. hierzu bereits Andreas Maurer/Didier Bigo/Penny Turnbull: Justice and Home Affairs and Democracy in the EU, in: Current Politics and Economics of Europe 3/2001, S. 313-349.

7 Vgl. Andreas Maurer/Jörg Monar: Parlamentarische Kontrolle und Innere Sicherheit im Prozeß der Europäisierung, in: Hans-Jürgen Lange (Hrsg.): Staat, Demokratie und Innere Sicherheit in Deutschland, Opladen 2000, S. 311-334. 
Beteiligung des Europäischen Parlaments im Rahmen das Mitentscheidungsverfahrens. ${ }^{8}$ Im Zuge der ,Implementierung ' des Amsterdamer Vertrages verzeichnet die Entscheidungsproduktion in der Justiz- und Innenpolitik der EU eine starke gesetzgeberische Dynamik vor allem in den Teilbereichen Visa-, Asyl- und Einwanderungspolitik die nunmehr zur ,Ersten Säule'gehören. Der weiterhin intergouvernmentale, von variierenden Rechts- und Polizeitraditionen geprägte Teilbereich der PJZS jedoch bleibt stark von Souveränitätsvorbehalten der Mitgliedstaaten und dem Einstimmigkeitszwang im Ministerrat geprägt. Der Fortschritt auf europäischer Ebene ist im Vergleich zu den anderen Teilbereichen begrenzt. Mit nunmehr 25, bald 27 Mitgliedstaaten ist die ohnehin schon langwierige und mühsame Konsensfindung im Ministerrat in der Dritten Säule noch schwieriger. ${ }^{9}$

Die von der Bevölkerung und den politischen Akteuren in den letzten Jahren wahrgenommene neue Sicherheitssituation war ein externer Anstoß dafür, die Terrorismus- und Kriminalitätsbekämpfung hoch auf die nationale und europäische politische Agenda zu rücken. Wichtige Fortschritte in der PJZS sind unter anderem der Auf- und Ausbau von Europol, die Einrichtung von Eurojust und die Schaffung des Instruments eines Europäischen Haftbefehls. ${ }^{10}$ Aus Sicht etlicher Mitgliedstaaten, allen voran Deutschland, die Beneluxstaaten und Österreich, besteht auch die Notwendigkeit die Zusammenarbeit zwischen den Strafverfolgungsbehörden der EU-Mitgliedstaaten zu verstärken. Die für die grenzüberschreitende Kriminalitätsbekämpfung daher zunehmend wichtige Überarbeitung der diesbezüglichen Regelungen im SDÜ ist unter den oben beschriebenen Bedingungen in der Dritten Säule schwierig. Zwar ersucht der Europäische Rat im Arbeitsprogramm für den Bereich der Innen- und Justizpolitik, dem so genannten Haager Programm, die Kommission, Vorschläge zur umfassenden Fortentwicklung des Schengen-Besitzstandes in Bezug auf die praktische polizeiliche Zusammenarbeit und den Informationsaustausch zwischen den mitgliedstaatlichen Strafverfolgungsbehörden zu unterbreiten. Das Gros der Vorschläge ist jedoch nur bedingt konsensfähig im Rat und kommt in den Verhandlungen zwischen den Mitgliedstaaten gar nicht oder nur mühsam voran. ${ }^{11}$ In der PJZS spielt daher die Kooperation unterhalb der EU-25-Schwelle - verkörpert durch ein engmaschiges Netzwerk bi- und multilateraler Kooperationsabkommen zwischen den Mitgliedstaaten der Union - eine wichtige Rolle.

Mit dem Vertrag von Prüm wird beabsichtigt diese Blockade auf europäischer Ebene zu durchbrechen. Der Vertrag sieht zwei Instrumente der Zusammenarbeit vor: erstens den vereinfachten Datenaustausch und zweitens die operative Zusammenarbeit der Polizei-, Strafverfolgungs- und Immigrationsbehörden. Im Wesentlichen geht es um folgendes:

8 Vgl. Jörg Monar: Ein Raum der Freiheit, der Sicherheit und des Rechts: Die Innen- und Justizpolitik nach Amsterdam, in: Mathias Jopp/Andreas Maurer/Otto Schmuck (Hrsg.): Die Europäische Union nach Amsterdam, Bonn 1998, S. 127-154; Jörg Monar: Justice and Home Affairs after Amsterdam: The treaty reforms and the challenge of their implementation, in: Jörg Monar/Wolfgang Wessels (Hrsg.): The European Union after the Treaty of Amsterdam, London/New York 2001, S. 267-295.

9 Vgl. Bericht des Ländervertreters im Ausschuss nach Artikel 36 EUV über die Beteiligung der Länder in Angelegenheiten der Europäischen Union im Jahr 2005, S. 6, Innenministerium Stuttgart, Az.: 3-0123.3-A36A, abrufbar unter: http://www.stmi.bayern.de/imperia/md/content/stmi/ministerium/imk/beschluesse/berichte/180 -_top_02_jb_05.pdf (letzter Zugriff: 19.06.2006).

10 Zur Entwicklung der PJZS vgl. Eugene Regan (Hrsg.): The New Third Pillar. Cooperation against Crime in the European Union, Dublin 2000.

11 Vgl. zuletzt den Vorschlag der Kommission für einen Beschluss des Rates ,zur Verbesserung der polizeilichen Zusammenarbeit der Mitgliedstaaten der Europäischen Union, vor allem an den Binnengrenzen, und zur Änderung des Übereinkommens zur Durchführung des Übereinkommens von Schengen“, $\operatorname{KOM}(2005) 317$, siehe Bericht des Ländervertreters, S. 23. 
- Die Signatarstaaten gewähren sich den gegenseitigen Online-Zugriff auf DNA- und Fingerabdruckdateien. So kann unter bestimmten Voraussetzungen künftig jeder Berechtigte in einem Vertragsstaat feststellen, ob zu einem bestimmten Fingerabdruck oder DNAIdentifizierungsmuster Daten in einem anderen Vertragsstaat gespeichert sind (Hit/No HitVerfahren). Im Falle eines Treffers werden diese (personenbezogenen) Daten auf dem Rechtshilfeweg übermittelt.

- Eigentümer-, Halter-, und Fahrzeugdaten aus den Fahrzeugregistern der jeweiligen Vertragsstaaten können gegenseitig automatisiert abgerufen werden.

- Im Rahmen von Großveranstaltungen und der Terrorismusbekämpfung können personenbezogene wie auch nicht-personenbezogene Daten zu präventiven Zwecken auch ohne Ersuchen einer Vertragspartei ausgetauscht werden.

- Zur Bekämpfung der illegalen Migration können so genannte Dokumentenberater in Staaten, die als Ausgangs- oder Transitländer illegaler Migration eingestuft werden, entsandt und dort unter anderem zu Beratungs- und Schulungszwecken eingesetzt werden.

- Zur Intensivierung der polizeilichen Zusammenarbeit sind gemeinsame Streifen und sonstige gemeinsame Einsatzformen zugelassen. Den auf fremdem Hoheitsgebiet eingesetzten Beamten können dabei hoheitliche Befugnisse eingeräumt werden.

- Schließlich ist der Einsatz von Flugsicherheitsbegleitern geregelt. ${ }^{12}$

Zwar reicht der Vertrag von Prüm nicht an die Regelungsbreite und -tiefe der bilateralen Verträge Deutschlands mit Österreich, den Niederlanden und der Schweiz heran, nach denen er in weiten Teilen modelliert ist, aber er geht in wichtigen Bereichen weit über den Vertragsrahmen von Schengen hinaus. Der Prümer Vertrag entfaltet somit eine wichtige Signalwirkung: Die sieben Prüm-Staaten bilden einen Kernraum innerhalb des Schengener EUNetzes, in dem polizeiliche Zusammenarbeit auf einem deutlich höheren Niveau stattfinden soll. Unter Effizienz- und Effektivitätsgesichtspunkten scheint der Fortschritt, der sich im Rahmen der Dritten Säule als äußerst schwierig entpuppt, über den intergouvernementalen ,Prümer Weg' einfacher erreichbar zu sein. Um diesen schließlich auf die gesamte EU zu übertragen, strebt der Vertrag den Beitritt einer maximalen Anzahl an EU-Mitgliedstaaten und schließlich die Überführung in den EU-Rechtsrahmen an. Die Signatarstaaten erklären bereits in der Vertragspräambel ihr Bestreben, mit diesem Vertrag, den sie in ihrer Funktion als EU-Mitgliedstaaten eingehen, den Integrationsprozess auf europäischer Ebene voranzutreiben. Ziel des Vertrages sei es, ,zur Fortentwicklung der Europäischen Zusammenarbeit [...] eine Vorreiterrolle bei der Erreichung eines möglichst hohen Standards in der Zusammenarbeit" einzunehmen. Artikel 1 VPgZ erlaubt den Beitritt aller EU-Mitgliedstaaten und sieht die regelmäßige Berichterstattung über den Fortschritt der Prümer Kooperation gegenüber der Kommission und dem Rat vor sowie eine Initiative zur Überführung des Vertragswerkes in den europäischen Rechtsrahmen spätestens drei Jahre nach Inkrafttreten. Welche Form diese Überführung konkret annehmen soll bleibt einstweilen offen.

\section{Parlamentarische Rückkopplung der, inneren Sicherheit‘ in der EU}

\section{Parlamentarische Begleitung des Schengen Prozesses}

Der Begriff ,innere Sicherheit“ ist den Europäischen Gemeinschaften und der Europäischen Union in ihrem spezifischen supranationalen Vertragskontext fremd und ist daher auch im konkreten Verlauf der Entwicklung innen- und justizpolitischer Politiken auf euro-

12 Vgl. Bericht des Ländervertreters, S. 16-17. 
päischer Ebene von weitgehender Unschärfe geprägt. Die späte, erst mit dem Maastrichter Vertrag allgemeinverbindlich sanktionierte Grundlegung einer intergouvernemental angelegten Innen- und Justizpolitik der $\mathrm{EU}^{13}$ erfolgte stattdessen über voneinander unabhängige, konzeptionelle Zugänge, ${ }^{14}$ in denen sich verschiedene Handlungsorientierungen und Leitbilder der europäischen Integration und damit auch unterschiedliche Konzeptionen bezüglich der parlamentarisch-demokratischen Rückbindung des angestrebten Policy-Prozesses widerspiegeln.

Einerseits förderten externe Faktoren wie die Zunahme terroristischer Aktivitäten in Westeuropa zu Beginn der 1970er Jahre engere politische Abstimmungen der Regierungen der EG-Mitgliedstaaten, den systematischen Informationsaustausch und Formen operativer polizeilicher Zusammenarbeit im Rahmen der TREVI-Kooperation. ,Innere Sicherheit“ war hier vor allem Element der intergouvernementalen Gefahrenabwehr in der EU. Die parlamentarische Rückkopplung dieser zwischenstaatlichen Prozesse war gering. ${ }^{15}$ Andererseits förderte das EG-Vertragsziel der Personenfreizügigkeit die Ausbildung einer europäischen Politik im Bereich der, inneren Sicherheit‘. Der zur Gewährleistung der Freizügigkeit notwendige Wegfall der Binnengrenzen erforderte eine Reihe von Ausgleichsmaßnahmen unter anderem im Bereich der Asyl- Immigrations-, und Visapolitik und eben auch der polizeilichen Zusammenarbeit. Aufgrund der Vorbehalte einer Reihe von Mitgliedstaaten erfolgten der Abbau der Binnengrenzen und die Etablierung dieser Ausgleichsmaßnahmen durch die Kooperation einiger EG-Mitgliedstaaten im Rahmen der Schengener Übereinkommen. ${ }^{16}$ Das SÜ klassifizierten die Signatarstaaten noch als bloße Absichtserklärung. Folglich sahen die Schengen-Mitglieder mit Ausnahme der Niederlande von einer Ratifizierung in den nationalen Parlamenten ab. Das SDÜ hingegen musste als internationales Übereinkommen von allen nationalen Parlamenten ratifiziert werden. Die Ratifikation des SDÜ verlieh aber nur einem Bruchteil des darin enthaltenen ,Acquis` eine formal-parlamentarische Legitimation, da die Implementierung des Übereinkommens nicht unmittelbar in die Inkraftsetzung und Anwendung aller hierin enthaltenen Maßnahmen und Garantien mündete. Aufgrund eines zweistufigen Inkraftsetzungsmechanismus, der ,die effektive völkerrechtliche Wirksamkeit des Abkommens von seiner rechtlichen Geltung abkoppelt", ${ }^{17}$ stellte sich die Frage bezüglich der parlamentarischen Rückbindung der durch den Schengener Exekutivausschuss (SEA) beschlossenen Maßnahmen zur Anwendung des Übereinkommens. Denn die nationalen Parlamente gaben mit der Ratifikation des SDÜ gesetzgeberische, in den Kernbestand der verfassungsmäßig verankerten Grund- und Menschenrechte eingreifende Befugnisse an ein intergouvernemental agierendes Durchführungsorgan ab, das seinerseits nicht durch ein parlamentarisches Gremium jenseits der nationalstaatlichen Ebene kontrolliert wurde. Auch wenn die Beschlüsse des SEA keine Übertragungen von Hoheitsbefugnissen darstellten und

13 Vgl. Peter-Christian Müller-Graff (Hrsg.): Europäische Zusammenarbeit in den Bereichen Justiz und Inneres. Der dritte Pfeiler der Europäischen Union, Baden-Baden 1996; Alexis Pauly (Hrsg.): De Schengen à Maastricht. Voie royale et course d'obstacles, Maastricht 1996.

14 Vgl. Wencelaus de Lobkowicz: Intergovernmental Co-operation in the Field of Migration - From the Single European Act to Maastricht, in: Jörg Monar/Roger Morgan: The Third Pillar of the European Union; Henri Etienne: The Commission of the European Community and Immigration, in: Gina D. Korella/Patrick M. Twomey (Hrsg.): Towards a European Immigration Policy, Brüssel 1995, S. 139-153.

15 Vgl. Andreas Maurer/Jörg Monar: Parlamentarische Kontrolle.

16 Vgl. Monica den Boer (Hrsg.): The Implementation of Schengen. First the Widening - Now the Deepening, Maastricht 1997.

17 Vgl. Raimund Schütz: Europaweite Freizügigkeit ohne demokratische Kontrolle? Überwachung der Anwendung der SDÜ-Konvention durch die nationalen Parlamente, in: Archiv des öffentlichen Rechts, Nr. 4/ 1995, S. 513. 
in der Regel einen nationalen Umsetzungsakt erforderten, griffen sie dennoch in die politikgestaltenden Zuständigkeiten der nationalen gesetzgeberischen Körperschaften ein.

Schütz ${ }^{18}$ kommt zu dem Ergebnis, dass sich die parlamentarisch-demokratische Rückbindung der SEA-Tätigkeiten in den meisten Mitgliedstaaten auf eine reine ex-post-Kontrolle beschränkte. In den Niederlanden, Italien, Belgien, Deutschland und Frankreich wurden jedoch besondere Prozeduren eingeführt. So verknüpfte das niederländische Parlament die Ratifizierung des SDÜ mit einer der Regierung auferlegten Bestimmung, jeden zur Entscheidung im SEA anstehenden Beschlussentwurf zu veröffentlichen und dem Parlament vorzulegen. Die Zustimmung des niederländischen Ministers im SEA stand seitdem unter einem parlamentarischen Zustimmungsvorbehalt, wobei von einer stillschweigenden Zustimmung auszugehen war, wenn nicht eine der beiden Kammern innerhalb eines Zeitraums von 14 Tagen nach Vorlage des Entwurfs explizit den Wunsch äußerte, diesen zu beraten und gegebenenfalls abzulehnen. Aufgrund dieser Bestimmung verfügte das niederländische Parlament zumindest formell über ein negatives Mitentscheidungsrecht im Eingriffsbereich des SDÜ. Ähnliche rechtlich verbindliche Parlamentsvorbehaltsrechte wurden im Rahmen der SDÜ-Ratifikation in Belgien und Italien vereinbart. Der Deutsche Bundestag wurde dagegen nur dann über Beschlussentwürfe des SEA vorab informiert, wenn die Transformation der SEA-Entscheidung seine legislativen Zuständigkeiten berührte. Ein Zustimmungsvorbehalt war jedoch nicht vorgesehen. Im Vergleich aller Schengen-Staaten war festzustellen, dass ,,sich durch die Einführung des Schengen-Systems die Möglichkeiten demokratischer Politikgestaltung in den Schengen-relevanten Bereichen verringert" hatten. ${ }^{19}$ Eine Kompensation dieses Rückgangs effektiver demokratischer Kontrolle durch das Europäische Parlament oder eine andere, der Schengener Mitgliedsstruktur angepasste parlamentarische Ebene, fand nicht statt.

\section{Prüm: Aus Schengen lernen oder eine Neuauflage parlamentsfreier Zusammenarbeit?}

Anlässlich eines Treffens der Justiz- und Innenminister der EU im Februar 2003 schlug der deutsche Innenminister seinen Amtskollegen aus den Benelux-Staaten und Frankreich vor, Verhandlungen zu einem multilateralen Vertrag zur Verstärkung der polizeilichen Zusammenarbeit aufzunehmen. In der Folge fanden zahlreiche Expertentreffen statt, aus denen sich Frankreich, aufgrund von Bedenken verfassungsrechtlicher Art zurückzog. Die Verhandlungspartner boten daraufhin Österreich die Teilnahme an den Verhandlungen an. Im Februar 2004 legte Deutschland einen ersten Vertragsentwurf vor, der in vier Expertentreffen und neun Verhandlungsrunden in Berlin verhandelt wurde. Begleitend fanden drei Ministertreffen statt. Anfang Mai 2005 erklärten dann auch Frankreich und Spanien ihre Bereitschaft, dem ausverhandelten Vertrag beizutreten. ${ }^{20}$

Aus demokratiepolitischer Sicht ist hinsichtlich dieses Verhandlungsprozesses folgendes zu bedenken. Wie bereits im Falle von Schengen, hatten weder die nationalen Parlamente, noch das Europäische Parlament noch die Vertreter der organisierten Zivilgesellschaft Kenntnis über oder gar Einfluss auf die Verhandlungen. Ausnahme ist das österreichische Parlament, welches von der Regierung über die Verhandlungen informiert wurde. Im Rahmen der Ratifikation haben die nationalen Parlamente jetzt nur noch die Möglichkeit der Zu-

18 Ebenda.

19 Ebenda, S. 534.

20 Vgl. Österreichischer Nationalrat: Beilagen zu den Stenographischen Protokollen des Nationalrates XXII. GP, 1155 - Staatsvertrag - Materialien, abrufbar unter: http://www.parlinkom.gv.at/pls/portal/docs/page/PG/DE/ XXII/I/I_01155-/fname_050447.pdf (letzter Zugriff: 19.06.2006). 
stimmung oder der Ablehnung. Letztere ist angesichts der Parlamentsmehrheiten allerdings unwahrscheinlich. Im deutschen Bundestag blieb im Verlaufe der schnellen Ratifizierung wenig Raum für öffentliche Debatten über einen Vertrag, dessen Regelungen unter Umständen tief in die Freiheitsrechte der Bürger eingreifen können. ${ }^{21}$ Wären die Prümer Regelungen innerhalb des EU-Rahmens verabschiedet worden, was aufgrund der mittlerweile bestehenden Rechtsgrundlagen in den EU-Verträgen möglich gewesen wäre, könnten die Parlamente zumindest über ihre Regierungen auf die Gesetzgebung einwirken. Auch die Zivilgesellschaft könnte sich in diesem Prozess einbringen.

Die Schengener Erfahrungen vor Augen erscheint auch das Mandat des im Prümer Vertrag vorgesehenen Ministerkomitees beratungsbedürftig. Möchten Parlamente verhindern, dass analog zum SEA rechtlich substantielle Beschlüsse gefasst werden, die, auf nationaler Ebene teils als Verwaltungsakte deklariert, nicht mehr der parlamentarischen Kontrolle unterstehen, dann sollte während der Ratifizierungsphase geklärt werden, welche Funktionen das Ministerkomitee und seine Beschlüsse übernehmen dürfen und wie nationale Parlamentskontrolle und -vorbehalte ausgestaltet werden könnten. Es finden sich im Vertrag keine Einzelermächtigungen für Umsetzungsbeschlüsse des Ministerkomitees - dies war beim SEA der Fall - und der Anwendungsbereich des Prümer Vertrages ist auch deutlich begrenzter im Vergleich zum Schengener Übereinkommen. Dennoch bleibt die Rolle des Ministerkomitees unklar. Ebenfalls klärungsbedürftig ist, welchen Inhalt und welche Rechtsform die im Vertrag vorgesehenen Durchführungsabkommen im nationalen Recht annehmen werden. In den drei Parlamenten, die bisher ratifiziert haben, wurde die zukünftige Möglichkeit der Einflussnahme analog zu Schengen verpasst. Es bleibt daher zu hoffen, dass die Parlamente die Entwicklungen des Prüm Acquis weiter verfolgen und gegebenenfalls zu einem späteren Zeitpunkt Kontrollmechanismen einrichten.

Das Hauptziel des Prümer Vertrages, der effizientere Informationsaustausch zum Zwecke der Kriminalitätsbekämpfung in einem Raum ohne Binnengrenzen, ist ein sehr wichtiges Vorhaben und wird von den Parlamenten der Vertragsstaaten nicht angezweifelt. Im Gegenteil, der Großteil der Parlamentarier unterstützt ausdrücklich das Ziel der effizienteren Kriminalitätsbekämpfung. Da Kompetenzerweiterungen der Polizeibehörden - innerstaatlich oder transnational - aus der Perspektive der Wahrung der Grund- und Bürgerrechte jedoch zu den besonders sensiblen Politikfeldern gehören, sollten Parlamente den Prümer Vertrag sehr gründlich behandeln. Dies müsste um so mehr der Fall sein, da seit den Terroranschlägen im Jahr 2001 in der öffentlichen Debatte das Spannungsverhältnisses von innerer Sicherheit und bürgerlichen Freiheitsrechten sehr zu Gunsten eines positiv besetzten Fokus auf den Aspekt der inneren Sicherheit diskutiert wird. ${ }^{22}$ Betrachtet man die Entwicklung des Raumes der Freiheit, der Sicherheit und des Rechts seit seiner ,Einführung' auf europäischer Ebene durch den Amsterdamer Vertrag, ist die Konzentration auf die Sicherheitsdimension offenkundig und der Aspekt ,eines gemeinsamen Raumes der (inneren) Sicherheit“ der am weitesten gediehene in dieser Trias. ${ }^{23}$

21 Deutscher Bundestag: Plenarprotokoll 16/36 vom 19. Mai 2006, abrufbar unter: http://dip.bundestag.de/btp/16/ 16036.pdf (letzter Zugriff: 19.06.2006).

22 Vgl. Andreas Maurer/Roderick Parkes: Democracy and European Justice and Home Affairs Policies under the shadow of September 11, in: Hans Günter Brauch/John Grin/Czeslaw Mesjasz u.a. (Hrsg.): Globalisation and Environmental Challenges: Reconceptualising Security in the 21st Century, Berlin/New York 2006 (i.E.).

23 Jörg Monar: Die politische Konzeption des Raumes der Freiheit, der Sicherheit und des Rechts: Vom Amsterdamer Vertrag zum Verfassungsentwurf des Konvents, in: Peter-Christian Müller-Graff (Hrsg.): Der Raum der Freiheit, der Sicherheit und des Rechts, Baden-Baden 2005, S. 29-41. 
Eine grund- und bürgerrechtlich inspirierte Debatte wert sind vor allem die ungeklärten Begrifflichkeiten in Artikel 14 und 16 VPgZ zum Austausch personenbezogener Daten bei der Verhinderung terroristischer Straftaten und Straftaten in Zusammenhang mit grenzüberschreitenden Großveranstaltungen sowie die weitgehende Kooperation im Bereich der DNA-Daten. Zur Verhinderung terroristischer Straftaten beispielsweise können die Vertragsparteien nach innerstaatlichem Recht untereinander personenbezogene Daten übermitteln, wenn bestimmte Tatsachen die Annahme rechtfertigen, dass die Betroffenen Straftaten nach Artikel 1 bis 3 des Rahmenbeschlusses 2002/475/JI des Rates zur Terrorismusbekämpfung begehen werden. Interpretationsoffen bleibt, was unter die „bestimmten Tatsachen“ fällt, die die „Annahme“ rechtfertigen, dass jemand eine terroristische Straftat begeht.

In Deutschland und Österreich fand im Rahmen der Ratifizierung in den Parlamentsgremien zumindest eine begrenzte, vor allem von der jeweiligen Opposition dominierte Debatte statt. Im Vordergrund stand die Beeinträchtigung bürgerlicher Freiheitsrechte, und dabei vor allem Aspekte bezüglich des Datenschutzes. Eine grundlegende, öffentliche Auseinandersetzung mit dem Thema blieb jedoch aus. Im spanischen Kongress wurde kein einziges Wort zum Vertrag verloren, der über eine Sammelliste rasant alle parlamentarischen Gremien bis zur Ratifizierung durchlief.

\section{Zielkonflikt Integrationsförderung versus Fragmentierung?}

Im Hinblick auf den Prüfmaßstab der Effizienz und Effektivität des europäischen Handlungsgefüges wirft Prüm politisch brisante Fragen auf: Dürfen, ja sollen die Prüm-Staaten auf der einen Seite explizit unter Verweis auf und unter dem Dach des EU-Rechts agieren, während sie auf der anderen Seite aber mit Blick auf die Kontrollrechte des EuGH, die Initiativrechte der Kommission, die Entscheidungsrechte des Europäischen Parlaments und die europapolitischen Mitwirkungsrechte der nationalen Parlamente außerhalb des EU-Rahmens handeln?

Mit dem neuen Vertrag etabliert eine Gruppe von Mitgliedstaaten ihre eigene Sicherheitsagenda im Hinblick auf die Regelung sehr sensibler in der EU primärrechtlich eindeutig normierter, aber in der Ausformulierung des Sekundärrechts oftmals strittiger Sachverhalte. Befürworter dieser ,Schengen-Vorgehensweise" sehen darin den Vorteil von Integrationsfortschritten gerade in einer Phase, in der die Vertiefung der EU nur sehr langsam voranschreitet, wie im Fall der PJZS. Vor dem Hintergrund der Probleme der Entscheidungsfindung in der Dritten Säule ist das Ausweichen auf diesen (intergouvernementalen) Weg der Integration. Jedoch haben sich seit der Unterzeichnung des Schengener Durchführungsübereinkommens vor mehr als 15 Jahren die Rahmenbedingungen für die Wiederholung derartiger Vertiefungsschritte grundlegend geändert, nämlich der Umfang des Acquis europäischer Innen- und Justizpolitik und die diesbezüglichen Entscheidungsstrukturen.

\section{Überschneidung mit und Vorwegnahme von EU-Maßnahmen?}

Mittlerweile existiert ein substanzieller Rechtskörper im Bereich der europäischen Innenund Justizpolitik. Anfang Mai 2006 umfasste dieser 1152 rechtsgültige Verordnungen, Richtlinien und sonstige Beschlüsse. Aus dieser Dichte ergeben sich potenziell Konflikte mit jedweder Form der verstärkten Zusammenarbeit. Alle drei von Prüm erfassten Bereiche - die Bekämpfung der grenzüberschreitenden Kriminalität, des Terrorismus und der illegalen Migration - werden mittlerweile auch durch EU-Recht geregelt. Die Prümer Regelungen müssen aufgrund dieses mittelbaren Bezugs daher immer vor dem Hintergrund des EU-Besitzstandes gesehen und bewertet werden. Kritiker mahnen in diesem Zusammenhang an, 
dass das in Artikel 10 EG-Vertrag normierte Loyalitätsprinzip verletzt werden könnte. ${ }^{24}$ Diesem Prinzip entsprechend sind die Mitgliedstaaten der Union darauf verpflichtet, alle Maßnahmen zur Erfüllung der EG-Vertragsverpflichtungen zu ergreifen und alle Maßnahmen zu unterlassen, die die Zielerfüllung des EG-Vertrages gefährden könnten. Zur Debatte steht somit, ob der Vertrag der Verwirklichung des Zieles der Schaffung eines europaweiten Raumes der Freiheit, der Sicherheit und des Rechts dient.

In den Bereichen des Datenaustausches und der polizeilichen Zusammenarbeit an den Grenzen nimmt der Prümer Vertrag zwei im Haager-Programm vorgesehene und gegenwärtig im Rat verhandelte Rahmenbeschlüsse vorweg (siehe Tabelle 1). Im Gegensatz zu den Prümer Regelungen würden diese EU-weit gelten. In der polizeilichen Zusammenarbeit geht Prüm über das auf EU-Ebene angestrebte Kooperationsniveau hinaus, beim Datenaustausch bleibt er dahinter zurück. Hier ist der Bewertungsmaßstab für die Prümer Regelungen die Bestrebung der EU-Ebene, den grenzüberschreitenden Datenaustausch in der Strafverfolgung durch den „Grundsatz der Verfügbarkeit“ zu erleichtern. Gemäß den Kommissionsvorstellungen erhalten die Behörden eines Mitgliedstaates grundsätzlich alle in einem anderen Mitgliedstaat verfügbaren und zur Strafverfolgung notwendigen Informationen. Die Suche nach relevanten Informationen erfolgt weitgehend automatisiert, das heißt über einen direkten Online-Zugriff auf die Datenbanken der anderen Mitgliedstaaten. Prüm ähnelt diesem Vorschlag. In beiden Regelwerken kann in einem ersten Verfahrensschritt nur auf anonymisierte Fundstellendaten zugegriffen werden. Für den zweiten Verfahrensschritt bleibt es in der Prümer Version den innerstaatlichen Rechtsvorschriften der dateiführenden Staaten vorbehalten, ob und wie diese anonymisierten Daten entschlüsselt und weitergegeben werden. Der Vorschlag für den EU-Rahmenbeschluss sieht dagegen vor, dass im Falle einer Übereinstimmung grundsätzlich der komplette Satz personenbezogener Daten auf Anfrage geliefert werden muss. Gleichzeitig definiert er aber für alle EU-Mitgliedstaaten einheitliche Gründe, auf deren Basis die Übermittlung personenbezogener Daten an andere Mitgliedstaaten verweigert werden kann. Dadurch, dass für den Informationsaustausch nicht mehr das nationale Recht maßgeblich ist, überwindet der Kommissionsvorschlag die Behinderung des Informationsaustausches zwischen den mitgliedstaatlichen Behörden. Prüm bleibt in diesem Punkt hinter den Ambitionen der Kommission zurück. Dieser Vorschlag für einen EU-Rahmenbeschluss liegt momentan im Ministerrat auf Eis. Zwei weitere von der Kommission für den Bereich des Austausches von DNA und Fingerabdrucksdaten angekündigte Vorschläge werden sich an den Prümer Regelungen orientieren. Ob der Vorschlag für einen umfassenden EU-Rahmenbeschluss zum Datenaustausch damit völlig obsolet ist, bleibt offen.

Die beiden Maßnahmen zur Bekämpfung der illegalen Migration - der Einsatz von Dokumentenberatern und die gemeinsame Rückführung von Drittstaatenangehörigen - bauen explizit auf bestehendes EU-Recht auf. Der Verweis erfolgt auf die Verordnung des Rates aus dem Jahre 2004, die den Aufbau eines Netzes von Verbindungsbeamten für Einwanderungsfragen regelt, die ihre Regierungen über die Situation in den Drittstaaten mit Blick auf Einwanderungsfragen, sowie Polizei- und Grenzkräfte der Länder in der Ausführung ihrer Tätigkeiten beraten sollen und ihre Kenntnisse untereinander austauschen. Diese Aufgaben sollen im Prüm-Rahmen die Dokumentenberater erfüllen. Nach Aussage der Signatarstaaten stellen die Prümer Dokumentenberater keine einfache Duplizierung der existierenden Strukturen dar, sondern eine Vertiefung der EU-Zusammenarbeit. Ob dies in der Praxis ohne Koordinierungsprobleme von statten geht bleibt abzuwarten. Auch die Prümer Regelungen zu gemeinsamen Rückführungen von Drittstaatsangehörigen überschneiden sich mit bereits

24 Vgl. dazu im Detail Thierry Balzacq: Security and the Two Level Game. 
geltenden EU-Rechtsakten in diesem Bereich und konterkarieren Bestrebungen auf europäischer Ebene, einheitliche Bedingungen für die Rückführungen über das Territorium mehrerer Mitgliedstaaten zu schaffen. Prüm bestätigt das Recht der Staaten, nationale Bedingungen für Rückführungen über ihre Staatsgebiet festzulegen und wird daher wohl eher die Effektivierung einer gemeinsamen Rückführungspraxis erschweren.

Der Vorwurf, dass die sieben Prüm-Staaten die 18 außen vor bleibenden EU-Staaten sowie die europäischen Entscheidungsmechanismen umgehen, um anstelle der Suche nach EU-weiten Kompromissen ihre eigenen Interessen effizienter durchzusetzen, ist angesichts dieser Befunde nicht einfach von der Hand zu weisen. Für eine Überführung in den EURechtsrahmen durch ein Protokoll zu den EG/EU-Verträgen à la Schengen bedürfte es der zum jetzigen Zeitpunkt unwahrscheinlichen Einstimmigkeit zwischen den Mitgliedstaaten. Die alternative Möglichkeit der Anbindung des Prümer Vertrages an den EU-Rechtsrahmen durch die in Art. 40 EUV vorgesehene verstärkte Zusammenarbeit wurde von der österreichischen Präsidentschaft als Ziel der Kooperation verworfen. ${ }^{25}$ Wahrscheinlicher ist schrittweise ,Infiltrierung ' der Verhandlungen auf europäischer Ebene durch das Prümer Vorbild, wie dies bereits im Falle des Datenaustausches sichtbar wird. ${ }^{26}$ Die Vertragsstaaten führen in diesem Zusammenhang an, dass, sobald zukünftig eine den Prümer Bereich betreffende EU-Regelung beschlossen wird, ,Prüm'-Recht hinter EU-Recht zurücktrete. Werden die Prümer Regelungen zum DNA-Datenaustausch oder der operativen Polizeikooperation wirklich hinfällig wenn zukünftige EU-Maßnahmen hierzu in Kraft treten, die womöglich hinter dem Prümer Kooperationsniveau zurückbleiben oder darüber hinausgehen? Oder bestehen die Prümer Regelungen in solchen Fällen dann parallel weiter? Wieso sollten die ,Prüm'-Staaten von vornherein im Rat EU-Regelungen zustimmen die hinter den nach ihren Präferenzen erstellten Prüm Regelungen zurückbleiben oder darüber hinausgehen? Dies ist nur wahrscheinlich, wenn sich die vorgesehenen EU-Maßnahmen mit den Prümer Maßnahmen in weiten Teilen decken. Eine solche Einigung zwischen 25 Mitgliedstaaten wird innerhalb der Dritten Säule schwierig zu erreichen sein. Dies erhöht die Gefahr, dass Einigungen nur in Verbindung mit Ausnahmeklauseln einzelner Staaten zustande kommen, wie Deutschland sie kürzlich im Falle des Rahmenbeschlusses über die Europäische Beweisanordnung in Anspruch genommen hat.

Insgesamt kann also nicht ausgeschlossen werden, dass Prümer- und EU- Regelungen zumindest partiell parallel fortbestehen und damit eine weitere Kooperationsebene und entsprechende Koordinationsprobleme in der europäischen Innen- und Justizpolitik eingeführt werden, auch wenn dies von den Signatarstaaten mit Verweis auf die Kollisionsklausel immer wieder verneint wird. Insbesondere im Fall der Vorwegnahme von angestrebten EU-Regelungen ist zu befürchten, dass Prüm die Einigung im Rat auf EU-weite Maßnahmen eher noch behindern wird. Auch wenn der Vertrag sein Ziel erfüllt und den Integrationsprozess durch eine Sogwirkung befördert, ist der Preis hierfür aller Wahrscheinlichkeit nach jedoch eine weitere Fragmentierung und höhere Komplexität der europäischen Innen- und Justizpolitik.

25 Vortrag der österreichischen Präsidentschaft, Sitzung des Ausschuss für Bürgerliche Freiheiten, Justiz und Inneres des Europäischen Parlaments am 4. Mai 2006.

26 Zum Datenschutz siehe Thilo Weicher: Wo liegt eigentlich Prüm? Der polizeiliche Datenaustausch in der EU bekommt eine neue Dimension, Datenschutz Nachrichten 1/2006, S. 12-15. 
Tabelle 1: Inhaltliche Überschneidungen des Vertrags von Prüm mit existierenden und derzeit verhandelten EU-Maßnahmen

\begin{tabular}{|c|c|c|}
\hline Titel EU-Maßnahme & $\begin{array}{l}\text { Rechts- } \\
\text { grundlage } \\
\text { EUV/EGV }\end{array}$ & Prüm Regelung \\
\hline \multicolumn{3}{|c|}{ Datenaustausch } \\
\hline $\begin{array}{l}\text { Vorschlag für einen Rahmenbeschluss des Rates } \\
\text { über den Austausch von Informationen nach dem } \\
\text { Grundsatz der Verfügbarkeit; KOM (2005) } 490\end{array}$ & $\begin{array}{l}\text { Art. 30(1)(b), } \\
\text { Art. 34(2)(b) } \\
\text { EUV }\end{array}$ & $\begin{array}{l}\text { Art. } 2-12 \\
\text { Austausch von DNA, daktylos- } \\
\text { kopischen und Fahrzeugregis- } \\
\text { terdaten }\end{array}$ \\
\hline $\begin{array}{l}\text { Vorschlag für einen Rahmenbeschluss des Rates } \\
\text { über den Schutz personenbezogener Daten, die } \\
\text { im Rahmen der polizeilichen und justiziellen Zu- } \\
\text { sammenarbeit in Strafsachen verarbeitet werden; } \\
\text { KOM(2005) } 475\end{array}$ & $\begin{array}{l}\text { Art. } 30, \\
\text { Art. } 31 \\
\text { Art. } 34(2)(b) \\
\text { EUV }\end{array}$ & $\begin{array}{l}\text { Kapitel } 7 \\
\text { Allgemeine Datenschutzbe- } \\
\text { stimmungen sowie Einzelbe- } \\
\text { stimmungen in den einzelnen } \\
\text { Kapiteln }\end{array}$ \\
\hline \multicolumn{3}{|c|}{ Bekämpfung der illegalen Migration } \\
\hline $\begin{array}{l}\text { Verordnung (EG) Nr. 377/2004 des Rates zur } \\
\text { Schaffung eines Netzes von Verbindungsbeam- } \\
\text { ten für Einwanderungsfragen }\end{array}$ & $\begin{array}{l}\text { Art. } 63(3)(b), \\
\text { Art. } 66 \mathrm{EGV}\end{array}$ & $\begin{array}{l}\text { Art. } 20-22 \\
\text { Einsatz von Dokumentenbera- } \\
\text { tern }\end{array}$ \\
\hline $\begin{array}{l}\text { Entscheidung des Rates 2004/573/EG betreffend } \\
\text { die Organisation von Sammelflügen zur Rück- } \\
\text { führung von Drittstaatsangehörigen, die individu- } \\
\text { ellen Rückführungsmaßnahmen unterliegen, aus } \\
\text { dem Hoheitsgebiet von zwei oder mehr Mitglied- } \\
\text { staaten }\end{array}$ & $\begin{array}{l}\text { Art. 63(3)(b) } \\
\text { EGV }\end{array}$ & $\begin{array}{l}\text { Art. } 23 \\
\text { Unterstützung bei Rückführun- } \\
\text { gen }\end{array}$ \\
\hline $\begin{array}{l}\text { Richtlinie 2003/110/EG des Rates über die Un- } \\
\text { terstützung bei der Durchbeförderung im Rah- } \\
\text { men von Rückführungsmaßnahmen auf dem } \\
\text { Luftweg }\end{array}$ & $\begin{array}{l}\text { Art. 63(3)(b) } \\
\text { EGV }\end{array}$ & $\begin{array}{l}\text { Art. } 23 \\
\text { Unterstützung bei Rückführun- } \\
\text { gen }\end{array}$ \\
\hline \multicolumn{3}{|c|}{ Polizeikooperation } \\
\hline $\begin{array}{l}\text { Vorschlag für einen Beschluss des Rates zur Ver- } \\
\text { besserung der polizeilichen Zusammenarbeit } \\
\text { zwischen den Mitgliedstaaten der EU, vor allem } \\
\text { an den Binnengrenzen, und zur Änderung des } \\
\text { Übereinkommens zur Durchführung des SDÜ } \\
\text { KOM(2005)317 }\end{array}$ & $\begin{array}{l}\text { Art. } 30 \\
(1)(a)(b)(c) \\
\text { Art. } 32 \\
\text { Art. } 34(2)(c) \\
\text { EUV }\end{array}$ & $\begin{array}{l}\text { Art. 24-32 } \\
\text { Gemeinsame Einsatzformen } \\
\text { (Streife, Nacheile, Wahrneh- } \\
\text { mung hoheitlicher Befugnisse } \\
\text { durch ausländische Beamte } \\
\text { etc.) und vieles mehr }\end{array}$ \\
\hline $\begin{array}{l}\text { Initiative des Königreichs der Niederlande im } \\
\text { Hinblick auf die Annahme des Beschlusses des } \\
\text { Rates über die Verstärkung der grenzüberschrei- } \\
\text { tenden polizeilichen Zusammenarbeit bei Groß- } \\
\text { veranstaltungen }(6930 / 2005)\end{array}$ & $\begin{array}{l}\text { Art. } 29,30,34 \\
\text { (2)(c) EUV }\end{array}$ & $\begin{array}{l}\text { Art. } 26 \\
\text { Hilfeleistungen bei Großereig- } \\
\text { nissen etc. }\end{array}$ \\
\hline
\end{tabular}




\section{Integration à la Prüm: Umgehung des institutionellen EU-Rahmens?}

Seit Schengen haben sich die Entscheidungsstrukturen auf der EU-Ebene tief greifend geändert. Das Europäische Parlament besitzt mittlerweile weitgehende Mitentscheidungsrechte in fast allen innen- und justizpolitischen Angelegenheiten. In der strafrechtlichen und polizeilichen Zusammenarbeit verfügen die Europaabgeordneten über umfassende Konsultationsrechte und Haushaltsbefugnisse. Sollte der Prümer Vertrag in der einen oder anderen Form in EU-Recht überführt werden, wären die Abgeordneten de facto mit Rechtsvorschriften konfrontiert, bei deren Erlass sie im EU-Rahmen Rechtsetzungsbefugnisse hätten geltend machen können. Auch das Initiativrecht der Kommission und die Kontrollbefugnisse des EuGH fielen bei dieser Art der ,nachholenden Integration “ unter den Tisch. Aufgefangen werden könnte diese Infragestellung des institutionellen Rahmens durch aktuelle Bemühungen im Kontext der europäischen Verfassungsvertragskrise, einzelnen Reformelementen des Vertrags über eine Verfassung für Europa auf der Grundlage der bestehenden Verträge vorzugreifen. Den Anfang hierzu machte Frankreichs Staatspräsident Chirac, der im Januar 2006 forderte, die EU ,,auf Basis der bestehenden Verträge“ zu reformieren um die Funktionsweise der Institutionen zu verbessern. Substanziell unterfüttert hat diese Initiative im April 2006 ein Schreiben der Außen- und Europaminister Frankreichs an die österreichische Außenministerin. Unter anderem schlugen sie die Nutzung der sogenannten Passerelle- bzw. Brücken-Klausel in Artikel 42 EUV vor, um die Überführung der Politiken aus der Dritten Säule des EU-Vertrages in den EG-Vertrag zu realisieren und damit die Vergemeinschaftung der polizeilichen und strafrechtlichen Zusammenarbeit voranzutreiben wie sie im Verfassungsvertrag bereits vorgesehen ist. Alle oder Teile der in der Dritten Säule normierten Politiken würden hierbei ins Verfahren der qualifizierten Mehrheit im Rat, vom einfachen Konsultationsrecht des Europäischen Parlaments zumindest mittelfristig zum Mitentscheidungsverfahren sowie zur Stärkung der gerichtlichen Kontrolle dieser Materien durch den Europäischen Gerichtshof übergehen. Auch die EU-Kommission und die finnische Ratspräsidentschaft haben sich dieser Linie angeschlossen. Die Aktivierung der Passerelle würde die Entscheidungsfindung in der PJZS deutlich vereinfachen und das Ausweichen der Regierungen auf Kooperationsformen außerhalb der Verträge à la Schengen und Prüm weniger attraktiv machen. Sie wäre allerdings kein unmittelbar greifender Rettungsanker der EU-Organe gegenüber Prüm. Der Prümer Vertrag sieht im Vorwort den möglichen Abschluss weiterer Verträge sowie die Beschlussfassung durch den Ministerausschuss vor und verstärkt damit Bedenken hinsichtlich der Etablierung eines umfassenden Besitzstandes analog zu ,Schengen', dessen Überführung in den EU-Rechtsrahmen rund 200, teilweise substanzielle Beschlüsse des Schengener Exekutivausschusses zu Tage brachte. ${ }^{27}$ Selbst wenn es bereits im zweiten Halbjahr 2006 gelänge, die Passerelle zu aktivieren, könnte eine mögliche Prümer Eigendynamik nur durch rasch folgende Umsetzungsmaßnahmen der EU in Anwendung der geänderten Verfahrensregeln abgemildert werden. Das Ziel der Integrationsförderung mag der Prümer Vertrag erreichen. Sehr wahrscheinlich jedoch wird, analog zu Schengen, der Preis für das Ausweichen in den kleinen, intergouvernementalen Rahmen eine weitere Fragmentierung dieses ohnehin schon stark fragmentierten Politikfeldes sein.

27 Vgl. Beschluss des Rates (1999/435/EG) vom 20. Mai 1999 zur Bestimmung des Schengen-Besitzstands zwecks Festlegung der Rechtsgrundlagen für jede Bestimmung und jeden Beschluss, die diesen Besitzstand bilden, nach Maßgabe der einschlägigen Bestimmungen des Vertrags zur Gründung der Europäischen Gemeinschaft und des Vertrags über die Europäische Union. 